\title{
A Cut-Free and Invariant-Free Sequent Calculus for PLTL *
}

\author{
J. Gaintzarain ${ }^{1}$, M. Hermo ${ }^{1}$, P. Lucio ${ }^{1}$, M. Navarro ${ }^{1}$, and F. Orejas ${ }^{2}$ \\ ${ }^{1}$ Dpto de Lenguajes y Sistemas Informáticos, Universidad del País Vasco, 20080-San \\ Sebastián, Spain. \\ ${ }^{2}$ Dpto de Lenguajes y Sistemas Informáticos, Universidad Politécnica de Catalunya, \\ 08034-Barcelona, Spain.
}

\begin{abstract}
Sequent calculi usually provide a general deductive setting that uniformly embeds other proof-theoretical approaches, such as tableaux methods, resolution techniques, goal-directed proofs, etc. Unfortunately, in temporal logic, existing sequent calculi make use of a kind of inference rules that prevent the effective mechanization of temporal deduction in the general setting. In particular, temporal sequent calculi either need some form of cut, or they make use of invariants, or they include infinitary rules. This is the case even for the simplest kind of temporal logic, propositional linear temporal logic (PLTL). In this paper, we provide a complete finitary sequent calculus for PLTL, called $\mathcal{F C}$, that not only is cut-free but also invariant-free. In particular, we introduce new rules which provide a new style of temporal deduction. We give a detailed proof of completeness.
\end{abstract}

\section{Introduction}

The development of automated deduction systems for temporal logic has followed two main proof-theoretical approaches: tableaux (see [12]) and resolution (see [1]), which are both refutational proof methods. Sequent calculi are usually used to provide a general deductive setting that uniformly embeds refutational methods and other deduction techniques such as goal-directed proofs or natural deduction. In temporal logic, tableaux methods generate graphs instead of the classical trees and resolution methods require more involved normal forms and inference rules than the classical clausal form and the classical resolution rule. This complicates the association of a sequent calculus proof to each tableaux graph or each resolution proof. In addition, existing sequent calculi for temporal logic (cf. $[6,8,11]$ ) make use of a kind of inference rules that prevents this correspondence and complicates the implementation of temporal deduction in the general setting. In particular, temporal sequent calculi either need some form of cut (classical cut or invariant-based cut) or they include infinitary rules. Cut rules imply the "invention" of lemmata, called cut formula, for their application. Invariants are particular cut formulas for proving temporal eventualities. This is the case even for the simplest kind of temporal logic, propositional linear temporal logic (PLTL). In this sense, the formulation of a cut-free, invariant-free finitary sequent calculus, can be considered a relevant open problem that is solved in this paper.

\footnotetext{
^ This work has been partially supported by Spanish Project TIN2004-079250-C03.
} 
More precisely, in [6] and [11], two sequent calculi for PLTL with invariant-based rules are presented. In fact, in both approaches, they present a system including also a cut rule and then prove cut elimination. However, invariant-based rules for temporal connectives cannot be avoided. In [8] various sequent calculi are presented for PLTL without the until operator (this means that the logic considered has a limited expressive power). He provides completeness and cut-elimination proofs, together with various interesting reductions among the various calculi. However, every calculus includes either some infinitary rule or some invariant-based rule. Other proof-theoretic approaches for PLTL include its first axiomatization á la Hilbert presented in [2], and the first detailed description of a tableaux method for deciding the satisfiability of any PLTL-formula presented in [12]. The satisfiability problem for PLTL is PSPACE-complete (cf. [10]). See [9] for a good survey about theorem-proving in PLTL and its extensions.

In this paper, we provide a complete finitary sequent calculus for PLTL, called $\mathcal{F C}$, that not only is cut-free but also invariant-free. In particular, we introduce a new rule for the until operator that provides a new style of temporal deduction for eventualities. Moreover, deduction for "always"-formulas is also affected by this new style.

In order to show completeness, we have not followed the standard approach of, first, proving completeness including a cut rule in the calculus and, then, showing a cut elimination result (cf. [3]). Actually, the first part of that approach, proving completeness of $\mathcal{F C}$ plus the cut rule, is quite easy. In particular, just with the rules in $\mathcal{F C}$ it is easy to derive every axiom (except the modus ponens rule) in the system proved complete in [5]. Obviously, with the addition of the cut rule one can easily derive modus ponens. Unfortunately, we have been unable to directly prove cut elimination. Instead, we have directly proved the completeness of $\mathcal{F C}$, which indirectly means that the cut rule is not needed. The proof is partially inspired by the tableaux method proposed in [5]. In particular their notion of maximal strongly connected components has been very useful in our proof. However, unlike [5], we use a filtration technique for constructing models from saturated consistent sets of formulas (as states).

The paper is organized as follows. Section 2 is a basic introduction to PLTL. In sections 3 and 4 we introduce our calculus $\mathcal{F C}$, proving its soundness. More precisely, in section 3 we describe the basic rules for describing the next $(\circ)$ and until $(\mathcal{U})$ connectives, while in section 4 we present some useful derived rules describing, in particular, the rest of the temporal connectives. Section 5 presents the completeness proof of $\mathcal{F C}$. Finally, in section 6 we draw some concluding remarks.

\section{PLTL: Language and Model Theory}

A PLTL-formula is built using the constant proposition $\mathbf{F}$, propositional variables (denoted by lowercase letters $p, q, \ldots$ ) from a set Prop, the classical connectives $\neg$ and $\vee$, and the temporal connectives $\circ$ and $\mathcal{U}$. A lowercase Greek letter $(\varphi, \psi, \chi, \gamma, \ldots)$ denotes a formula and an uppercase one $(\Phi, \Delta, \Gamma, \Psi, \Omega, \ldots)$ denotes a finite set of PLTL-formulas. PLTL-formulas of the form $p$ and $\neg p$, where $p \in$ Prop, are called literals and PLTL-formulas that do not begin with the connective $\neg$ are called positive. As usual other connectives can be defined in terms of the previous ones: $\mathbf{T} \equiv \neg \mathbf{F}$, 
$\varphi \wedge \psi \equiv \neg(\neg \varphi \vee \neg \psi), \diamond \varphi \equiv \mathbf{T} \mathcal{U} \varphi, \square \varphi \equiv \neg \diamond \neg \varphi$. PLTL-formulas of the form $\varphi \mathcal{U} \psi$ and $\diamond \varphi$ are called eventualities. In the rest of this paper, we simply say formula instead of PLTL-formula. The operator next translates any set of formulas into another (possibly empty) set of formulas next $(\Phi)=\{\varphi \mid \circ \varphi \in \Phi\}$.

It is well known that PLTL is a non-compact logic. As a consequence, strong completeness requires an infinitary proof system, whose deduction rules may require infinitely many premises. Our calculus is finitary, hence, as usual (see, e.g. [6], [2] and [11]), our completeness result is in this sense, weak. Therefore, along this paper, every set of PLTL-formulas is assumed to be finite. Given any (finite) set $\Phi=\left\{\varphi_{1}, \ldots, \varphi_{n}\right\}$ we will use $\Phi\urcorner$ to denote the formula $\neg \varphi_{1} \vee \ldots \vee \neg \varphi_{n}$. In particular, $\left.\Phi\right\urcorner$ is the constant F when $\Phi$ is empty.

Definition 1. A PLTL-structure $\mathcal{M}$ is a pair $\left(S_{\mathcal{M}}, V_{\mathcal{M}}\right)$ such that $S_{\mathcal{M}}$ is a denumerable sequence of states $s_{0}, s_{1}, s_{2}, \ldots$ and $V_{\mathcal{M}}$ is a map $V_{\mathcal{M}}: S_{\mathcal{M}} \rightarrow 2^{\text {Prop }}$.

Intuitively, $V_{\mathcal{M}}$ specifies which atomic propositions are (necessarily) true in each state.

Definition 2. The truth of a formula $\varphi$ in the state $s_{j}$ of a PLTL-structure $\mathcal{M}$, which is denoted by $\langle\mathcal{M}, j\rangle=\varphi$, is inductively defined as follows:

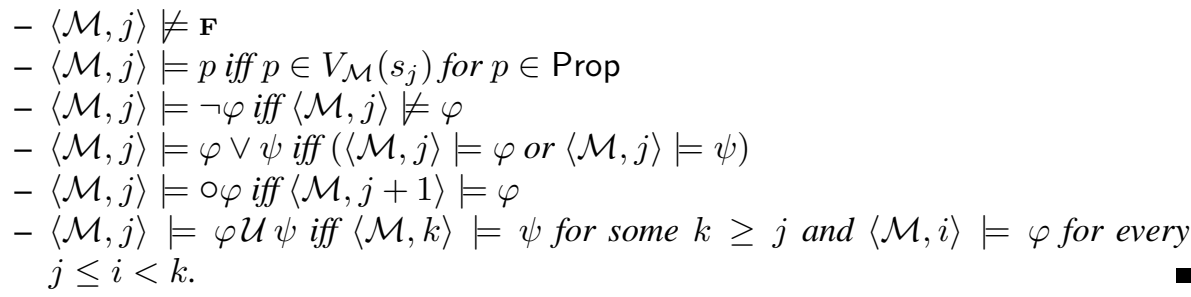

This is extended to sets in the usual way: $\langle\mathcal{M}, j\rangle=\Phi$ iff $\langle\mathcal{M}, j\rangle \mid=\varphi$ for all $\varphi \in \Phi$. We say that $\mathcal{M}$ is a model of $\Phi$, in symbols $\mathcal{M} \mid=\Phi$, iff $\langle\mathcal{M}, 0\rangle \mid=\Phi$. A satisfiable set of PLTL-formulas has at least one model, otherwise it is unsatisfiable. The logical consequence relation between a set of formulas $\Phi$ and a formula $\chi$, denoted as $\Phi=\chi$, is defined in the following way:

$$
\begin{gathered}
\Phi \models \chi \text { iff for every PLTL-structure } \mathcal{M} \text { and every } j \in \mathbb{N}: \\
\qquad \text { if }\langle\mathcal{M}, j\rangle \models \Phi \text { then }\langle\mathcal{M}, j\rangle \models \chi
\end{gathered}
$$

\section{The Sequent Calculus $\mathcal{F C}$}

In this section, we introduce a sound and complete sequent calculus, called $\mathcal{F C}$, that is fully free of cut. That is, in $\mathcal{F C}$ there are neither classical cut rules nor invariant-based rules for temporal connectives. The calculus $\mathcal{F C}$ uses asymmetric sequents, i.e. sequents formed by a set of assumptions and a single conclusion. The former set is called the antecedent of the sequent and the latter formula is called the consequent. We write $\Delta \vdash \chi$ to represent a sequent whose antecedent is $\Delta$ and whose consequent is $\chi$. We have preferred to formulate the calculus by means of asymmetric (or one-conclusion) 
Classical connectives rules

\begin{tabular}{|ccccc|}
\hline & $\Delta, \varphi \vdash \chi$ \\
$(\neg L) \frac{\Delta \vdash \varphi}{\Delta, \neg \varphi \vdash \chi}$ & $(R \neg) \frac{\Delta, \varphi \vdash \mathbf{F}}{\Delta \vdash \neg \varphi}$ & $(\vee L) \frac{\Delta, \psi \vdash \chi}{\Delta, \varphi \vee \psi \vdash \chi}$ & $(R \vee) \frac{\Delta \vdash \varphi}{\Delta \vdash \varphi \vee \psi}$ & $\frac{\Delta \vdash \psi}{\Delta \vdash \varphi \vee \psi}$ \\
\hline
\end{tabular}

Temporal connectives rules

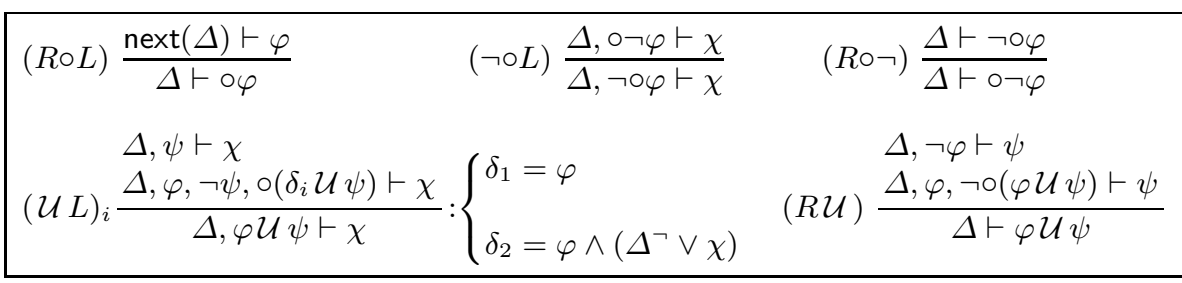

Structural rules

\begin{tabular}{llll}
$(A s) \Delta, \varphi \vdash \varphi$ & $(W k) \frac{\Delta \vdash \chi}{\Delta, \Delta^{\prime} \vdash \chi}$ & $(C d) \frac{\Delta, \neg \varphi \vdash \mathbf{F}}{\Delta \vdash \varphi}$ & (оF) $\frac{\Delta \vdash \text { ०F }}{\Delta \vdash \chi}$ \\
\hline
\end{tabular}

Fig. 1. The sequent calculus $\mathcal{F C}$

sequents, instead of symmetric (multiple-conclusioned) sequents, because the former are closer to natural deduction and captures better our intuition in logical reasoning. A multiple-conclusioned system can be easily obtained from $\mathcal{F C}$. For getting rid of some rules and giving a more compact presentation, we could also take the one-sided sequent approach (also known as Tait-style). However, it requires to keep formulas in negation normal form and results a bit more unusual and unnatural at first sigth.

The calculus $\mathcal{F C}$ consists of the primitive rules that are summarized in Fig. 1. We have split these rules into three packages. Two of them consist of rules for classical and temporal connectives, respectively. These rules follow the traditional style of introduction of the connective in the left/right part of the sequent. In addition we need some structural rules which form the third package.

The rules for classical connectives are classical. With respect to the temporal connectives, the three rules for the next operator, $(R \circ L),(\neg \circ L)$ and $(R \circ \neg)$, are well known in the literature of PLTL. Besides, by means of $(\mathcal{U} L)_{i}$ we represent two rules for two different $\delta_{i}$ where $i=1$ or $i=2$. The rules $(\mathcal{U} L)_{1}$ and $(R \mathcal{U})$ are also well known. Both are included in the existing Gentzen systems where other invariant-based rules for the until operator are given (cf.[6, 11]). Instead, we add a rule $(\mathcal{U} L)_{2}$ which does not require invariant generation. This rule $(\mathcal{U} L)_{2}$, which up to our knowledge is completely new, can be considered quite peculiar, since the second premise includes a formula which depends on the whole conclusion of the rule. ${ }^{3}$ In addition $(\mathcal{U} L)_{2}$ leads to a new deduction style that is opposite, in some sense, to the invariant-based reasoning. The underlying idea in the rule $(\mathcal{U} L)_{2}$ is that the sequences of states along which the satisfaction of an eventuality is delayed should be ever-changing sequences. In the

\footnotetext{
${ }^{3}$ Remember that $\Delta$ is always assumed to be a finite set and that $\left.\Delta\right\urcorner$ is $\mathbf{F}$ whenever $\Delta$ is empty.
} 
proof of the soundness theorem, we show in detail that the rule $(\mathcal{U} L)_{2}$ is correct. We believe that this correctness proof reflects the intuition behind the rule.

Regarding structural rules, $\left(\mathbf{O F}_{\mathbf{F}}\right)$ is the only rule that is not a classical rule. At first sight, the introduction of the weakening rule $(W k)$ in the structural package could be surprising since very commonly $(W k)$ is an elementary property and an admissible rule. However, the form of the rule $(\mathcal{U} L)_{2}$ prevents that traditional methods for proving admissibility (cf. [7]) could be applied to the calculus $\mathcal{F C}$. Although experimental work (see Example 6) indicates that $(W k)$ could be admissible in $\mathcal{F C}$, this is still an interesting open problem. This work is mainly focused in completeness, the minimality of the calculus remains as future work.

An $\mathcal{F C}$-proof is a tree (written right side up, with its root on the bottom) labelled with sequents. The sequent to be proved labels its root, the leaves are labelled with axioms (which are rules without premises), and all the local subtrees must be accepted by some inference rule in $\mathcal{F C}$. In the Examples 4 and 5, we give a sequence of sequents that ends with the root (the proved sequent) and add additional information for describing the structure of the tree.

The expression $\Gamma \vdash_{\mathcal{F C}} \chi$ is used to denote that there exists an $\mathcal{F C}$-proof of the sequent $\Gamma \vdash \chi$. We say that a set of formulas $\Gamma$ is $\mathcal{F C}$-consistent if and only if $\Gamma \forall_{\mathcal{F C}} \mathbf{F}$. The soundness of $\mathcal{F C}$ means that every $\mathcal{F C}$-provable sequent, namely $\Gamma \vdash \chi$, is correct regarding to logical consequence. In particular, every satisfiable set of formulas is $\mathcal{F C}$ consistent.

Theorem 3. For any set offormulas $\Gamma \cup\{\chi\}$, if $\Gamma \vdash_{\mathcal{F C}} \chi$ then $\Gamma \models \chi$.

Proof. By induction on the length of the $\mathcal{F C}$-proof, it suffices to prove that every primitive rule of $\mathcal{F C}$ (see Fig. 1) is correct in the sense of preserving the logical consequence relation between the antecedent and the consequent.

Now, the correctness proof of most rules is just routine. Actually, the only correctness proof that poses some difficulties is the proof of the rule $(\mathcal{U} L)_{2}$. Hence, we only give the details for this rule.

We will show that, if we assume that $\Delta \cup\{\varphi \mathcal{U} \psi, \neg \chi\}$ is satisfiable, then we would build a countermodel for some of the two premises of the rule $(\mathcal{U} L)_{2}$. Let $\langle\mathcal{M}, i\rangle \models$ $\Delta \cup\{\varphi \mathcal{U} \psi, \neg \chi\}$ and $s_{1}$ the least $s \geq i$ such that $\langle\mathcal{M}, s\rangle \models \psi$. If $s_{1}=i$ then $\langle\mathcal{M}, i\rangle$ serves as countermodel for the first premise. Otherwise, if $s_{1}>i$, let $s_{2}$ be the greatest $s$ such that $i \leq s<s_{1}$ and $\langle\mathcal{M}, s\rangle \models \Delta \cup\{\varphi \mathcal{U} \psi, \neg \chi\}$. As a consequence of the choice of $s_{1}$ and $s_{2}$, it holds $\left\langle\mathcal{M}, s_{2}\right\rangle \models \mathrm{o}\left(\left(\varphi \wedge\left(\Delta^{\urcorner} \vee \chi\right)\right) \mathcal{U} \psi\right)$. Then, $\left\langle\mathcal{M}, s_{2}\right\rangle$ is a countermodel of the second premise.

\section{Derived Rules and Proofs}

In this section we present some derived rules that can be used as a shortcut for several lines of primitive-rules-only proofs. Actually, some of these rules are used below in the proof of the completeness theorem.

The first group of derived rules, including the contraposition rules $(C p 1)$ and $(C p 2)$, can be derived in a standard way from the classical primitive rules in $\mathcal{F C}$. 


$$
\begin{array}{lll}
(C p 1) \frac{\Delta, \neg \varphi \vdash \psi}{\Delta, \neg \psi \vdash \varphi} & (C p 2) \frac{\Delta, \varphi \vdash \psi}{\Delta, \neg \psi \vdash \neg \varphi} & (\mathbf{F} L) \Delta, \mathbf{F} \vdash \chi \\
(C d L) \Delta, \varphi, \neg \varphi \vdash \chi & (\neg \neg L) \frac{\Delta, \varphi \vdash \chi}{\Delta, \neg \neg \varphi \vdash \chi} & (\neg \vee L) \frac{\Delta, \neg \varphi, \neg \psi \vdash \chi}{\Delta, \neg(\varphi \vee \psi) \vdash \chi}
\end{array}
$$

For the temporal connectives, the following derived rules will be used later:

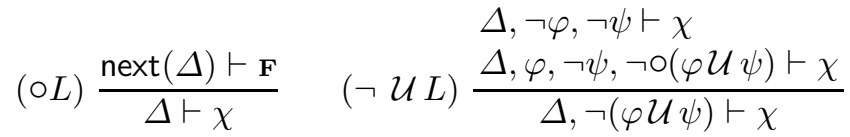

It is easy to check that $(\circ L)$ is derived by $(R \circ L)$ and $(\circ \mathbf{F})$ and $(\neg \mathcal{U} L)$ by $(C p 1)$ and $(R \mathcal{U})$.

Other derived rules allow us to reason about the rest of the classical or temporal connectives, which have been introduced as a shorthand to abbreviate some formulas. For instance, since $\varphi \wedge \psi$ stands for $\neg(\neg \varphi \vee \neg \psi)$, the classical sequent rules for $\wedge$ can be derived:

$$
(\wedge L) \frac{\Delta, \varphi, \psi \vdash \chi}{\Delta, \varphi \wedge \psi \vdash \chi} \quad(R \wedge) \frac{\Delta \vdash \varphi \Delta \vdash \psi}{\Delta \vdash \varphi \wedge \psi}
$$

Likewise, using the abbreviations $\diamond \varphi$ and $\square \varphi$ for $\mathbf{T} \mathcal{U} \varphi$ and $\neg \diamond \neg \varphi$, respectively, we are also able to derive the following useful rules:

$$
\begin{aligned}
& (\diamond L)_{i} \frac{\Delta, \neg \vdash, \chi\left(\delta_{i} \mathcal{U} \varphi\right) \vdash \chi}{\Delta, \diamond \varphi \vdash \chi}:\left\{\begin{array}{l}
\delta_{1}=\mathbf{T} \\
\left.\delta_{2}=\Delta\right\urcorner \vee \chi
\end{array} \quad(R \diamond) \frac{\Delta, \neg \circ \diamond \varphi \vdash \varphi}{\Delta \vdash \diamond \varphi}\right. \\
& (\square L) \frac{\Delta, \varphi, \circ \square \varphi \vdash \chi}{\Delta, \square \varphi \vdash \chi} \quad(R \square)_{i} \frac{\Delta, \circ\left(\delta_{i} \mathcal{U} \neg \varphi\right) \vdash \neg \varphi}{\Delta \vdash \square \varphi}:\left\{\begin{array}{l}
\delta_{1}=\mathbf{T} \\
\left.\delta_{2}=\Delta\right\urcorner
\end{array}\right.
\end{aligned}
$$

Note also that, by $(\square L)$ and $(\neg \circ L)$, the following contradiction rule is also derivable:

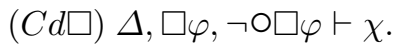

It is well known that the until operator $\mathcal{U}$ is not expressible in temporal logic with only $\circ, \square$, and $\diamond$ as temporal operators (cf. [4,2]). As a consequence a complete calculus for the sublogic that uses $\diamond$ instead of $\mathcal{U}$ cannot be derived (by abbreviation) from $\mathcal{F C}$, since the rule $(\diamond L)_{2}$ needs the until operator for expressing its second premise.

Let us now illustrate the $\mathcal{F C}$-style of natural reasoning by means of some examples of $\mathcal{F C}$-proofs. In order to allow easier reading, we have underlined, at each step, the formulas that are related with the applied deduction rule. 
Example 4. The following proof shows that $p, \square(\neg p \vee \circ p) \vdash_{\mathcal{F C}} \square p$. This is a typical property of induction on time. We have used $\square \varphi$ to abbreviate $\square(\neg p \vee \circ p)$.

$$
\begin{aligned}
& \text { 1. }-\underline{p}, \square \varphi \vdash \underline{p} \text { by }(A s) \\
& \text { 2. }-\underline{p}, \square \varphi, \underline{\neg p}, \neg \neg p, \circ((\neg p \vee \neg \square \varphi) \mathcal{U} \neg p) \vdash \mathbf{F} \quad \text { by }(C d L) \\
& \text { 3. }-p, \underline{\square \varphi, \neg \square \varphi}, \neg \neg p, \circ((\neg p \vee \neg \square \varphi) \mathcal{U} \neg p) \vdash \mathbf{F} \quad \text { by }(C d L) \\
& \text { 4. }-\underline{p}, \square \varphi, \neg \underline{p} \vdash \mathbf{F} \quad \text { by }(C d L) \\
& \text { 5. }-\underline{p}, \square \varphi, \neg p \vee \neg \square \varphi, \neg \neg p, \circ((\neg p \vee \neg \square \varphi) \mathcal{U} \neg p) \vdash \mathbf{F} \quad \text { by } 2,3 \text { and }(\vee L) \\
& \text { 6. }-p, \square \varphi, \underline{(\neg p \vee \neg \square \varphi) \mathcal{U} \neg p} \vdash \mathbf{F} \quad \text { by } 4,5 \text { and }(\mathcal{U} L)_{1} \\
& \text { 7. }-p, \underline{\neg p}, \circ \square \varphi, \circ((\neg p \vee \neg \square \varphi) \mathcal{U} \neg p) \vdash \underline{\neg p} \text { by }(A s) \\
& \text { 8. }-p, \underline{\circ p, \circ \square \varphi, \circ((\neg p \vee \neg \square \varphi) \mathcal{U} \neg p)} \vdash \neg p \quad \text { by } 6 \text { and }(\circ L) \\
& \text { 9. }-p, \neg p \vee \circ p, \circ \square \varphi, \circ((\neg p \vee \neg \square \varphi) \mathcal{U} \neg p) \vdash \neg p \quad \text { by } 7,8 \text { and }(\vee L) \\
& \text { 10. }-p, \underline{\square}, \circ((\neg p \vee \neg \square \varphi) \mathcal{U} \neg p) \vdash \neg p \quad \text { by } 9 \text { and }(\square L) \\
& \text { 11. }-p, \square \varphi \vdash \square p \text { by } 1,10 \text { and }(R \square)_{2} \text {. }
\end{aligned}
$$

It is worthy to note that $\{\square \beta, \circ((\varphi \vee \neg \square \beta) \mathcal{U} \psi)\}$ and $\{\square \beta, \circ(\varphi \mathcal{U} \psi)\}$ are equivalent sets of formulas. As a consequence, the above proof could be simplified if the sequent to be derived at step 10 were $p, \square \varphi, \circ(\neg p \mathcal{U} \neg p) \vdash \neg p$ instead of

$$
p, \square \varphi, \bigcirc((\neg p \vee \neg \square \varphi) \mathcal{U} \neg p) \vdash \neg p .
$$

A practical implementation of $\mathcal{F C}$ should apply the rules $(\mathcal{U} L)_{2}$ (and also $(\square L)_{2}$ and $\left.(\diamond L)_{2}\right)$ yielding as subgoal $\circ(\varphi \mathcal{U} \psi)$ instead of $\circ((\varphi \vee \neg \square \beta) \mathcal{U} \psi)$. In general, the rule $(\mathcal{U} L)_{2}$ should take into account the equivalence of the following two sets of formulas:

$$
\{\square \alpha, \neg(\alpha \mathcal{U} \beta), \circ((\varphi \vee(\alpha \mathcal{U} \beta)) \mathcal{U} \psi)\} \text { and }\{\square \alpha, \neg(\alpha \mathcal{U} \beta), \circ(\varphi \mathcal{U} \psi)\}
$$

Note that the former pair of equivalent sets is a particular case of the latter one.

Example 5. The following is an $\mathcal{F C}$-proof of the sequent $p \mathcal{U} q, \neg q \vdash \circ \diamond q$ :

$$
\begin{aligned}
& \text { 1. }-\underline{q, \neg q} \vdash \circ \diamond q \text { by }(\mathrm{CdL}) \\
& \text { 2. }-\underline{q}, \neg \circ \diamond q \vdash \underline{q} \text { by (As) } \\
& \text { 3. }-p, \underline{\circ \diamond q}, \neg q, \circ((p \wedge(\neg \neg q \vee \circ \diamond q)) \mathcal{U} q), \underline{\neg \circ \diamond q} \vdash q \text { by }(\mathrm{CdL}) \\
& \text { 4. }-p, \neg \neg q, \neg q, \circ((p \wedge(\neg \neg q \vee \circ \diamond q)) \mathcal{U} q), \neg \circ \diamond q \vdash q \text { by }(\mathrm{CdL}) \\
& \text { 5. }-p, \neg \neg q \vee \circ \diamond q, \neg q, \circ((p \wedge(\neg \neg q \vee \circ \diamond q)) \mathcal{U} q), \neg \circ \diamond q \vdash q \text { by } 3,4 \text { and }(\vee L) \\
& \text { 6. }-\underline{p \wedge(\neg \neg q \vee \circ \diamond q)}, \neg q, \circ((p \wedge(\neg \neg q \vee \circ \diamond q)) \mathcal{U} q), \neg \circ \diamond q \vdash q \text { by } 5 \text { and }(\wedge L) \\
& \text { 7. }-(p \wedge(\neg \neg q \vee \circ \diamond q)) \mathcal{U} q, \neg \circ \diamond q \vdash q \text { by } 2,6 \text { and }(\mathcal{U} L)_{1} \\
& \text { 8. }-(p \wedge(\neg \neg q \vee \diamond \diamond q)) \mathcal{U} q \vdash \underline{ } \underline{q} \text { by } 7 \text { and }(R \diamond) \\
& \text { 9. }-p, \neg q, \underline{\circ((p \wedge(\neg \neg q \vee \circ \diamond q)) \mathcal{U} q)} \vdash \underline{\circ \diamond q} \text { by } 8 \text { and }(R \circ L) \\
& \text { 10. }-\underline{p \mathcal{U} q}, \neg q \vdash \circ \diamond q \text { by } 1,9 \text { and }(\mathcal{U} L)_{2}
\end{aligned}
$$


It is easy to check that using only the rule $(\mathcal{U} L)_{1}$ we cannot prove the sequent.

Example 6. Consider the sequent $q, p \mathcal{U} \mathbf{F} \vdash \mathbf{F}$. It is easy to give an $\mathcal{F C}$-proof of $p \mathcal{U} \mathbf{F} \vdash$ $\mathbf{F}$ since by $(\mathcal{U} L)_{2}$ it should be proved $\mathbf{F} \vdash \mathbf{F}$ and $p, \neg \mathbf{F}, \circ(p \wedge(\mathbf{F} \vee \mathbf{F})) \mathcal{U} \mathbf{F} \vdash \mathbf{F}$. The latter is easily proved by $(R \circ L)$ and $(\mathcal{U} L)_{1}$. Finally, by $(W k), q, p \mathcal{U} \mathbf{F} \vdash \mathbf{F}$ is derived from $p \mathcal{U}_{\mathbf{F}} \vdash \mathbf{F}$.

It could be believed that $(W k)$ is essential for proving this kind of sequents, where some part of the antecedent is unnecessary for entailing the consequent. However, the following is a scketch of an $\mathcal{F C}$-proof of the sequent $q, p \mathcal{U} \mathbf{F} \vdash \mathbf{F}$ that does not use the rule $(W k)$ :

The first two main goals are: $q, \mathbf{F} \vdash \mathbf{F}$ and $q, p, \neg \mathbf{F}, \circ((p \wedge(\neg q \vee \mathbf{F})) \mathcal{U} \mathbf{F}) \vdash \mathbf{F}$. The former is an instance of $(A s)$, while the latter reduces to

$$
(p \wedge(\neg q \vee \mathbf{F})) \mathcal{U} \mathbf{F} \vdash \mathbf{F}
$$

by $(\circ \mathbf{F})$ and $(R \circ L)$. From this, by $(\mathcal{U} L)_{2}$ and $(\wedge L)$, we obtain two new goals. The first is $\mathbf{F} \vdash \mathbf{F}$, which is an $(A s)$. The second goal is

$$
p, \neg q \vee \mathbf{F}, O((p \wedge(\neg q \vee \mathbf{F}) \wedge(\mathbf{F} \vee \mathbf{F})) \mathcal{U} \mathbf{F}) \vdash \mathbf{F}
$$

Then,

$$
(p \wedge(\neg q \vee \mathbf{F}) \wedge(\mathbf{F} \vee \mathbf{F})) \mathcal{U} \mathbf{F} \vdash \mathbf{F}
$$

is obtained by $(\mathrm{OF})$ and $(R \circ L)$. Finally, $(\mathcal{U} L)_{1},(\wedge L)$ and $(A s)$ suffice.

This $(W k)$-free deduction style can be easily generalized to any sequent of the form $\Delta, \varphi \mathcal{U}_{\mathbf{F}} \vdash \mathbf{F}$, since the maximum number of nested next operators in $\Delta, \varphi$ is finite. In fact, we conjecture that $(W k)$ is admissible in $\mathcal{F C}$.

\section{The Completeness of $\mathcal{F C}$}

In this section, we prove that $\mathcal{F C}$ is a complete calculus using the technique of filtration. In particular, we define a notion of saturated set of formulas that enables the construction of a model for any set of formulas $\Phi$ such that $\Phi \forall \mathcal{F C}$ F. To this end, we first build a nondeterministic structure in which this model is embedded. The idea of using maximal strongly connected components, inspired by [5], is crucial in handling eventualities in this nondeterministic structure.

In the first subsection, we introduce a notion of saturation for sets of formulas which preserves $\mathcal{F C}$-consistency. In the second subsection, we show how to associate a nondeterministic structure to any $\mathcal{F C}$-consistent set of formulas. Finally, we prove the completeness of the calculus $\mathcal{F C}$.

\subsection{Saturated Sets of Formulas}

The closure of a set of formulas $\Phi$ consists of all formulas that we may use for constructing a model of $\Phi$. 
Definition 7. Let $\Phi$ be a set of formulas. Let subform $(\Phi)$ be the set of all the subformulas of the formulas in $\Phi$. Let basic $(\Phi)=\operatorname{subform}(\Phi) \cup\{\neg \varphi \mid \varphi \in \operatorname{subform}(\Phi)\}$. The closure set of $\Phi$, denoted $\mathrm{clo}(\Phi)$, is the extension of basic $(\Phi)$ with the following two sets offormulas:

$$
\begin{gathered}
\{\circ(\varphi \mathcal{U} \psi), \neg \circ(\varphi \mathcal{U} \psi), \circ \neg(\varphi \mathcal{U} \psi) \mid \varphi \mathcal{U} \psi \in \operatorname{basic}(\Phi)\} \\
\{\circ \neg \varphi \mid \neg \circ \varphi \in \operatorname{basic}(\Phi)\} .
\end{gathered}
$$

For example, if $\Phi$ is the singleton $\{p \wedge(p \mathcal{U} \neg \circ q)\}$ then clo $(\Phi)$ consists of the union of the following four sets:

$$
\begin{gathered}
\{p \wedge(p \mathcal{U} \neg \circ q), p, p \mathcal{U} \neg \circ q, \neg \circ q, \circ q, q\} \\
\{\neg(p \wedge(p \mathcal{U} \neg \circ q)), \neg p, \neg(p \mathcal{U} \neg \circ q), \neg \neg \circ q, \neg q\} \\
\{\circ(p \mathcal{U} \neg \circ q), \neg \circ(p \mathcal{U} \neg \circ q), \circ \neg(p \mathcal{U} \neg \circ q)\} \\
\{\circ \neg q\}
\end{gathered}
$$

where the first set is subform $(\Phi)$, whose joint with the second set constitutes basic $(\Phi)$. The last two sets respectively correspond with the two final extensions in the above definition.

Now, we define a successor relation on sets of formulas.

Definition 8. Let $\Omega_{1}$ and $\Omega_{2}$ be two subsets of clo $(\Phi)$ for some set $\Phi$. We say that $\Omega_{2}$ is a $\Phi$-successor of $\Omega_{1}$ iff $\varphi \in \Omega_{2}$ for all $\circ \varphi \in \Omega_{1}$. The set of $\Phi$-successors of a given set of formulas $\Omega$ is

$$
\operatorname{succ}_{\Phi}(\Omega)=\left\{\Omega^{\prime} \subseteq \operatorname{clo}(\Phi) \mid \Omega^{\prime} \text { is a } \Phi \text {-successor of } \Omega\right\} .
$$

Definition 9. We say that a set $\Omega$ offormulas is saturated iff it satisfies the following conditions:

1. If $\varphi \vee \psi \in \Omega$ then $\varphi \in \Omega$ or $\psi \in \Omega$

2. If $\neg(\varphi \vee \psi) \in \Omega$ then $\neg \varphi \in \Omega$ and $\neg \psi \in \Omega$

3. If $\varphi \mathcal{U} \psi \in \Omega$ then $\psi \in \Omega$ or $\{\varphi, \neg \psi, \circ(\varphi \mathcal{U} \psi)\} \subseteq \Omega$

4. If $\neg(\varphi \mathcal{U} \psi) \in \Omega$ then $\{\neg \psi, \neg \varphi\} \subseteq \Omega$ or $\{\varphi, \neg \psi, \neg \circ(\varphi \mathcal{U} \psi)\} \subseteq \Omega$

5. If $\neg \neg \varphi \in \Omega$ then $\varphi \in \Omega$.

6. If $\neg \circ \varphi \in \Omega$ then $\circ \neg \varphi \in \Omega$.

Given a set $\Phi$, we denote by $\operatorname{satur}(\Phi)$ the set of all saturated subsets of clo $(\Phi)$. For any $\Gamma \subseteq \operatorname{clo}(\Phi)$, we denote by $\operatorname{satur}^{\Gamma}(\Phi)$ the subset of satur $(\Phi)$ that includes all the supersets of $\Gamma$. In particular, satur $(\Phi)=\operatorname{satur}^{\emptyset}(\Phi)$ where $\emptyset$ denotes the empty set.

For the additionally defined connectives, the saturation conditions are easily deduced from Definition 9.

Proposition 10. The saturation conditions for $\wedge, \diamond$ and $\square$ are:

- If $\varphi \wedge \psi \in \Omega$ then $\varphi \in \Omega$ and $\psi \in \Omega$

- If $\neg(\varphi \wedge \psi) \in \Omega$ then $\neg \varphi \in \Omega$ or $\neg \psi \in \Omega$ 
- If $\diamond \varphi \in \Omega$ then $\varphi \in \Omega$ or $\{\neg \varphi, \diamond \diamond \varphi\} \subseteq \Omega$

- If $\neg \diamond \varphi \in \Omega$ then $\{\neg \varphi, \neg 0 \diamond \varphi\} \subseteq \Omega$

- If $\square \varphi \in \Omega$ then $\{\varphi, \circ \square \varphi\} \subseteq \Omega$

- If $\neg \square \varphi \in \Omega$ then $\{\varphi, \neg 0 \square \varphi\} \subseteq \Omega$ or $\neg \varphi \in \Omega$.

Note that if $\Phi$ is finite so is $\operatorname{clo}(\Phi)$. As a consequence, every $\Omega \in \operatorname{satur}(\Phi)$ is also finite.

The following lemma states that any subset of a $\mathcal{F C}$-consistent set can be extended to a saturated set while preserving the consistency of the whole set.

Lemma 11. For all sets of formulas $\Phi, \Psi, \Gamma$ such that $\Gamma \subseteq \operatorname{clo}(\Phi)$ and $\Gamma, \Psi \forall_{\mathcal{F C}} \mathbf{F}$, there exists at least one $\widehat{\Gamma} \in \operatorname{satur}^{\Gamma}(\Phi)$ such that $\widehat{\Gamma}, \Psi \forall_{\mathcal{F C}} \mathbf{F}$.

Proof. Suppose that $\widehat{\Gamma}, \Psi \vdash_{\mathcal{F C}} \mathbf{F}$ for all $\widehat{\Gamma} \in \operatorname{satur}^{\Gamma}(\Phi)$. Then, a $\mathcal{F C}$-proof of $\Gamma, \Psi \vdash \mathbf{F}$ can be easily built using these sequents as leaves and the rules $(\vee L),(\neg \vee L),(\mathcal{U} L)_{1}$, $(\neg \mathcal{U} L),(\neg \neg L)$ and $(\neg \circ L)$.

Note that $\Psi$ (in the above lemma) is not required to be a subset of the closure of $\Phi$. It could be seen as the context of $\Gamma$ and, in particular, it could be empty.

Corollary 12. If $\Phi \forall_{\mathcal{F C}} \mathbf{F}$ then there exists $\Omega \in \operatorname{satur}^{\Phi}(\Phi)$ such that $\Omega \forall_{\mathcal{F C}} \mathbf{F}$.

\subsection{Nondeterministic Models of $\mathcal{F C}$-Consistent Sets}

We are going to build a model whose states are $\mathcal{F C}$-consistent saturated sets. We use the following notion of nondeterministic PLTL-structure for representing collections of PLTL-structures. In fact, each infinite path in a nondeterministic PLTL-structure is a PLTL-structure.

Definition 13. $A$ nondeterministic PLTL-structure (nd-PLTL-structure, for short) $\mathcal{G}$ is a triple $\left(S_{\mathcal{G}}, R_{\mathcal{G}}, V_{\mathcal{G}}\right)$ such that:

- $S_{\mathcal{G}}$ is a finite non-empty set of states

- $R_{\mathcal{G}} \subseteq S_{\mathcal{G}} \times S_{\mathcal{G}}$ is called reachability relation

- $V_{\mathcal{G}}$ is a map $V_{\mathcal{G}}: S_{\mathcal{G}} \rightarrow 2^{\text {Prop. }}$

A path $\pi$ in a nd-PLTL-structure $\mathcal{G}$ is a non-empty sequence of states $s_{0}, s_{1}, \ldots \in S_{\mathcal{G}}$ and $s_{i} \in R_{\mathcal{G}}\left(s_{i-1}\right)$ for all $i \geq 1$.

We denote by $R_{\mathcal{G}}^{+}$and $R_{\mathcal{G}}^{*}$ the transitive closure and the reflexive-transitive closure of the reachability relation $R_{\mathcal{G}}$, respectively.

Definition 14. The truth of a formula $\varphi$ in a state $s$ of a nd-PLTL-structure $\mathcal{G}$, denoted by $\langle\mathcal{G}, s\rangle \models \varphi$, is defined as in the Definition 2, except for the temporal operators:

- $\langle\mathcal{G}, s\rangle \models \circ \varphi$ iff for all $s^{\prime} \in R_{\mathcal{G}}(s)\left\langle\mathcal{G}, s^{\prime}\right\rangle=\varphi$

- $\langle\mathcal{G}, s\rangle \models \varphi \mathcal{U} \psi$ iff there exists a finite path $s_{0}, s_{1}, \ldots, s_{n}$ in $S_{\mathcal{G}}$ such that $s=s_{0}$, $\left\langle\mathcal{G}, s_{n}\right\rangle=\psi$ and $\left\langle\mathcal{G}, s_{i}\right\rangle=\varphi$ for every $0 \leq i \leq n-1$. 
Note that, the above satisfaction definition of $\mathcal{U}$ only requires the existence of a path because nd-PLTL-structures could contain infinite paths that repeat infinitely many times a subsequence of states and do not reach some other finitely reachable states.

Now, we associate a nondeterministic structure to any consistent set.

Definition 15. For any given $\mathcal{F C}$-consistent set of formulas $\Phi, \mathcal{G}_{\Phi}=\left(S_{\mathcal{G}_{\Phi}}, R_{\mathcal{G}_{\Phi}}, V_{\mathcal{G}_{\Phi}}\right)$ is the nd-PLTL-structure where

- $S_{\mathcal{G}_{\Phi}}=\left\{\Omega \mid \Omega \in \operatorname{satur}(\Phi)\right.$ and $\left.\Omega \forall \forall_{\mathcal{F C}} \mathbf{F}\right\}$

- $\Omega^{\prime} \in R_{\mathcal{G}_{\Phi}}(\Omega)$ iff $\Omega^{\prime} \in \operatorname{succ}_{\Phi}(\Omega)$ for all $\Omega, \Omega^{\prime} \in S_{\mathcal{G}_{\Phi}}$

- $V_{\mathcal{G}_{\Phi}}(\Omega)=\{p \mid p \in \Omega$ and $p \in \operatorname{Prop}\}$.

Note that, according to Corollary $12, S_{\mathcal{G}_{\Phi}}$ cannot be empty. In the rest of this section we will assume that $\Phi$ is always an $\mathcal{F C}$-consistent set of formulas and $\mathcal{G}_{\Phi}$ is its associated nd-PLTL-structure. Now, we will show how the notion of maximal strongly connected components [5] yields a partition in $S_{\mathcal{G}_{\Phi}}$.

Definition 16. A strongly connected component (scc, for short) is a subset $\mathcal{S}$ of $S_{\mathcal{G}_{\Phi}}$ such that every pair formed by two different states $\Omega_{1}, \Omega_{2} \in \mathcal{S}$ satisfies that $\Omega_{2} \in$ $R_{\mathcal{G}_{\Phi}}^{+}\left(\Omega_{1}\right)$ and $\Omega_{1} \in R_{\mathcal{G}_{\Phi}}^{+}\left(\Omega_{2}\right)$.

A maximal scc (mscc, for short) is an scc $\mathcal{S}$ such that there is no scc $\mathcal{S}^{\prime} \subseteq S_{\mathcal{G}_{\Phi}}$ that satisfies $\mathcal{S} \subsetneq \mathcal{S}^{\prime}$.

We will denote by $[\Omega]$ the mscc where $\Omega$ is included and $\Leftrightarrow$ is the binary relation induced by $R_{\mathcal{G}_{\Phi}}$ as follows:

$\left[\Omega_{1}\right] \Leftrightarrow\left[\Omega_{2}\right]$ iff there exist $\Omega_{1}^{\prime} \in\left[\Omega_{1}\right], \Omega_{2}^{\prime} \in\left[\Omega_{2}\right]$ such that $\Omega_{2}^{\prime} \in R_{\mathcal{G}_{\Phi}}\left(\Omega_{1}^{\prime}\right)$.

Note that an mscc $[\Omega]$ could consist just of the state $\Omega$. In such case $[\Omega]$ can represent (on its own) a model only when $\Omega \in R_{\mathcal{G}_{\Phi}}(\Omega)$. An mscc that consists of exactly one state $\Omega$ such that $\Omega \notin R_{\mathcal{G}_{\Phi}}(\Omega)$ is called trivial. Otherwise, we say that it is a nontrivial $\operatorname{mscc}$ (nt-mscc, for short).

Definition 17. A path $\pi=\Omega_{0}, \Omega_{1}, \ldots$ in $S_{\mathcal{G}_{\Phi}}$ is fulfilling iffor every $\Omega_{i} \in \pi$ and every $\varphi \mathcal{U} \psi \in \Omega_{i}$ there exists some $j \geq i$ such that $\psi \in \Omega_{j}$ and for every $i \leq k \leq j-1$, $\varphi \in \Omega_{k}$.

An scc $\mathcal{S}$ in $S_{\mathcal{G}_{\Phi}}$ is self-fulfilling if for every $\Omega \in \mathcal{S}$ and every formula $\varphi \mathcal{U} \psi \in \Omega$, there exists a finite path $\Omega_{0}, \Omega_{1}, \ldots, \Omega_{n}$ in $\mathcal{S}$ such that $\Omega_{0}=\Omega, \psi \in \Omega_{n}$ and $\varphi \in \Omega_{i}$ for every $0 \leq i \leq n-1$.

Lemma 18. For every $\Omega \in S_{\mathcal{G}_{\Phi}}$ the set $R_{\mathcal{G}_{\Phi}}(\Omega)$ is non-empty.

Proof. If $\Omega \in S_{\mathcal{G}_{\Phi}}$ then $\Omega \forall \forall_{\mathcal{F C}}$ F. Hence next $(\Omega) \forall \forall_{\mathcal{F C}} \mathbf{F}$ holds by rules $(R \circ L)$ and (OF). From Lemma 11 there exists at least one $\Omega^{\prime} \in S_{\mathcal{G}_{\Phi}}$ such that $\Omega^{\prime} \in \operatorname{succ}_{\Phi}(\Omega)$.

Corollary 19. For every $\Omega \in S_{\mathcal{G}_{\Phi}}$ there is at least one infinite path $\Omega_{0}, \Omega_{1}, \ldots$ such that $\Omega=\Omega_{0}$.

Now, we will show that $\mathcal{G}_{\Phi}$ satisfies, by construction, the adequate properties for handling eventualities. In particular, in the next proposition we show that non-satisfied eventualities are kept in paths at least until they are fulfilled. 
Proposition 20. Let $\Omega \in S_{\mathcal{G}_{\Phi}}$ such that $\varphi \mathcal{U} \psi \in \Omega$. For every finite path $\Omega_{0}, \Omega_{1}, \ldots, \Omega_{n}$ in $S_{\mathcal{G}_{\Phi}}$ such that $\Omega_{0}=\Omega$ and every $1 \leq i \leq n$ : if $\varphi \mathcal{U} \psi \notin \Omega_{i}$ then $\psi \in \Omega_{k}$ for some $0 \leq k<i$ and $\varphi \in \Omega_{j}$ for all $0 \leq j \leq k-1$.

Proof. By induction on $\mathrm{n}$. The case $n=0$ trivially holds. For $n \geq 1$, we distinguish the following cases. First, if either $i=n$ and there exists $j \leq n-1$ such that $\varphi \mathcal{U} \psi \notin \Omega_{j}$ or $1 \leq i<n$, then the property holds by the induction hypothesis. Second, if $i=n$ and $\varphi \mathcal{U} \psi \in \Omega_{j}$ for all $0 \leq j \leq n-1$, then $\psi \in \Omega_{j}$ or $\{\varphi, \neg \psi, \circ(\varphi \mathcal{U} \psi)\} \subseteq \Omega_{j}$, since each $\Omega_{j}$ is saturated. This implies that $\psi \in \Omega_{n-1}$ because otherwise $\circ(\varphi \mathcal{U} \psi) \in \Omega_{n-1}$ which would mean $\varphi \mathcal{U} \psi \in \Omega_{n}$.

The next proposition shows how negated eventualities propagate in $\mathcal{G}_{\Phi}$.

Proposition 21. Let $\Omega \in S_{\mathcal{G}_{\Phi}}$ such that $\neg(\varphi \mathcal{U} \psi) \in \Omega$. Then, every finite path $\pi=$ $\Omega_{0}, \Omega_{1}, \ldots, \Omega_{n}$ in $S_{\mathcal{G}_{\Phi}}$ such that $\Omega_{0}=\Omega$ satisfies one of the two following properties:

(a) $\{\varphi, \neg \psi, \neg(\varphi \mathcal{U} \psi)\} \subseteq \Omega_{i}$ for any $i \in\{0 . . n\}$

(b) There exists $0 \leq j \leq n$ such that $\{\neg \varphi, \neg \psi\} \subseteq \Omega_{j}$ and $\{\varphi, \neg \psi, \neg(\varphi \mathcal{U} \psi)\} \subseteq \Omega_{i}$ for any $i \in\{0 . . j-1\}$.

Proof. By induction on $n$. Since $\Omega$ is saturated, the case $n=0$ is trivial. For $n \geq 1$, the induction hypothesis guarantees that the path $\pi^{\prime}=\Omega_{0}, \Omega_{1}, \ldots, \Omega_{n-1}$ satisfies one of the properties (a) or (b). If $\pi^{\prime}$ satisfies (b), so does $\pi$. If $\pi^{\prime}$ satisfies (a) then by definition of $S_{\mathcal{G}_{\Phi}}$ we have $\{\varphi, \neg \psi, \neg(\varphi \mathcal{U} \psi)\} \subseteq \Omega_{n}$ or $\{\neg \varphi, \neg \psi\} \subseteq \Omega_{n}$. Hence, $\pi$ verifies (a) or (b) respectively.

Now we will prove that for any $\Omega \in S_{\mathcal{G}_{\Phi}}$, either the mscc $[\Omega]$ is a self-fulfilling nt-mscc or there exists a self-fulfilling nt-mscc that is reachable from $[\Omega]$.

Lemma 22. For any non-self-fulfilling mscc $[\Omega]$ in $S_{\mathcal{G}_{\Phi}}$, there exists (at least) one $\Omega^{\prime} \in$ $S_{\mathcal{G}_{\Phi}}$ such that $\Omega^{\prime} \notin[\Omega]$ and $[\Omega] \Leftrightarrow\left[\Omega^{\prime}\right]$.

Proof. For a trivial mscc, this is an easy consequence of Lemma 18. Hence, we assume $[\Omega]$ to be a nt-mscc which is not self-fulfilling. That is, there is some $\Omega_{0} \in[\Omega]$ and some formula $\varphi \mathcal{U} \psi \in \Omega_{0}$ such that there does not exist a finite path $\Omega_{0}, \Omega_{1}, \ldots, \Omega_{n}$ in $[\Omega]$ such that $\psi \in \Omega_{n}$ and $\varphi \in \Omega_{i}$ for every $0 \leq i<n$. Then, for all $\Delta \in[\Omega]$ :

$$
\{\varphi, \neg \psi, \varphi \mathcal{U} \psi, \bigcirc(\varphi \mathcal{U} \psi)\} \subseteq \Delta
$$

Let us consider the subset of $S_{\mathcal{G}_{\Phi}}$ formed by all the states that are successors of some state in $[\Omega]$ :

$$
\mathcal{S}([\Omega])=\bigcup_{\Delta \in[\Omega]} R_{\mathcal{G}_{\Phi}}(\Delta)
$$

Since $[\Omega]$ is a nt-mscc it must verify $[\Omega] \subseteq \mathcal{S}([\Omega])$. If $[\Omega] \subsetneq \mathcal{S}([\Omega])$ the lemma holds trivially. On the contrary, if $[\Omega]=\mathcal{S}([\Omega])$ we show that there is a contradiction as follows. Consider any state $\Delta \in[\Omega] \subseteq S_{\mathcal{G}_{\Phi}}$. Since $\Delta$ is $\mathcal{F C}$-consistent, then $\Delta \forall_{\mathcal{F C}}$ F. Hence, by rules $(\mathcal{U} L)_{2}$ and $(\neg L)$, we have that

$$
\Delta, \circ\left(\left(\varphi \wedge \Delta^{\urcorner}\right) \mathcal{U} \psi\right) \forall \mathcal{F C} \mathbf{F}
$$


Hence, by $(R \circ L)$, the set next $(\Delta) \cup\left\{\left(\varphi \wedge \Delta^{\urcorner}\right) \mathcal{U} \psi\right\}$ is also $\mathcal{F C}$-consistent. Then, by Lemma 11 , there exists at least one set $\Delta^{\prime} \in \operatorname{satur}^{\text {next }(\Delta)}(\Phi)$ such that $\Delta^{\prime},(\varphi \wedge$ $\left.\Delta^{\urcorner}\right) \mathcal{U} \psi \forall_{\mathcal{F C}}$ F. By $(W k), \Delta^{\prime}$ is also $\mathcal{F C}$-consistent. Hence, $\Delta^{\prime} \in S_{\mathcal{G}_{\Phi}}$ and, by construction $\Delta^{\prime} \in R_{\mathcal{G}_{\Phi}}(\Delta) \subseteq \mathcal{S}([\Omega])$. Therefore, $\Delta^{\prime} \in[\Omega]$, since we are supposing that $[\Omega]=\mathcal{S}([\Omega])$. It is worthy to note that $R_{\mathcal{G}_{\Phi}}(\Delta)$ should be non-empty by Lemma 18 . Besides, since $\neg \psi \in \Delta^{\prime}$ (by construction) and $\Delta^{\prime},\left(\varphi \wedge \Delta^{\urcorner}\right) \mathcal{U} \psi \forall \nvdash_{\mathcal{F C}} \mathbf{F}$, the rules $(\mathcal{U} L)_{2}$ and $(C d L)$ allow us to conclude that

$$
\left.\Delta^{\prime}, \varphi \wedge \Delta^{\urcorner}, O\left(\left(\varphi \wedge \Delta^{\urcorner} \wedge\left(\Delta^{\prime}\right)\right\urcorner\right) \mathcal{U} \psi\right) \forall \mathcal{F C} \mathbf{F}
$$

Hence, by $(W k)$, we have obtained from $\Delta$ an $\mathcal{F C}$-consistent set $\Delta^{\prime}$ such that $\Delta^{\prime} \cup$ $\left.\left\{\circ\left(\left(\varphi \wedge \Delta^{\urcorner} \wedge\left(\Delta^{\prime}\right)\right\urcorner\right) \mathcal{U} \psi\right)\right\}$ is also $\mathcal{F C}$-consistent. Starting with any $\Delta_{0} \in[\Omega]$ and repeating the above procedure we can construct a path $\pi=\Delta_{0}, \Delta_{1}, \ldots$ of states in $[\Omega]$ such that for every $i \geq 1$

$$
\Delta_{i},\left(\varphi \wedge \Delta_{0}^{\urcorner} \wedge \Delta_{1}^{\urcorner} \wedge \ldots \wedge \Delta_{i-1}^{\urcorner}\right) \mathcal{U} \psi \forall \mathcal{F C}_{\mathcal{C}} \mathbf{F}
$$

By finiteness of $[\Omega]$, there must exist $n \geq 1$ such that $\Delta_{n}=\Delta_{i}$ for some $0 \leq i \leq n-1$. In particular, for such $n$ we have that

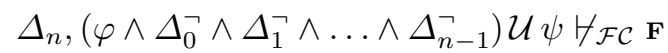

But this is a contradiction, by $(\mathcal{U} L)_{1},(\wedge L)$ and $(W k)$, because $\Delta_{n}, \Delta_{n}^{\neg} \vdash_{\mathcal{F C}} \mathbf{F}$ can be easily derived using $(\vee L)$ and $(C d L)$.

Corollary 23. For any $\Omega \in S_{\mathcal{G}_{\Phi}}$, either the mscc $[\Omega]$ is a self-fulfilling nt-mscc or there exists $\Omega^{\prime} \in S_{\mathcal{G}_{\Phi}}$ such that $\Omega^{\prime} \notin[\Omega], \Omega^{\prime} \in R_{\mathcal{G}_{\Phi}}^{+}(\Omega)$ and $\left[\Omega^{\prime}\right]$ is a self-fulfilling nt-mscc.

Proof. By finiteness of $S_{\mathcal{G}_{\Phi}}$, if $[\Omega]$ is not a self-fulfilling non-trivial mscc, then Lemma 22 guarantees the existence of $\left[\Omega^{\prime}\right]$. In the case of a trivial mscc, also Lemma 18 should be used.

Lemma 24. (Nondeterministic Model Existence) For every $\Omega \in S_{\mathcal{G}_{\Phi}}$ it holds that if $\varphi \in \Omega$ then $\left\langle\mathcal{G}_{\Phi}, \Omega\right\rangle \models \varphi$.

Proof. By structural induction on $\varphi$. The case of literals is trivial by definition of $\mathcal{G}_{\Phi}$. For formulas of the form $\neg \neg \varphi, \varphi \vee \psi, \neg(\varphi \vee \psi), \circ \varphi$ and $\neg \circ \varphi$ it holds by definition of $\mathcal{G}_{\Phi}$ and the induction hypothesis on $\{\varphi\},\{\varphi, \psi\},\{\neg \varphi, \neg \psi\},\{\varphi\}$ and $\{\neg \varphi\}$, respectively.

For $\varphi \mathcal{U} \psi$, by the above Proposition 20 and Corollary 23 there exists a finite path $\Omega_{0}, \Omega_{1} \ldots \Omega_{n}$ in $S_{\mathcal{G}_{\Phi}}$ such that $\Omega_{0}=\Omega, \psi \in \Omega_{n}$ and $\varphi \in \Omega_{i}$ for every $0 \leq i \leq n-1$. By the induction hypothesis, $\left\langle\mathcal{G}_{\Phi}, \Omega_{n}\right\rangle \models \psi$ and $\left\langle\mathcal{G}_{\Phi}, \Omega_{i}\right\rangle \models \varphi$ for every $0 \leq i \leq n-1$ and consequently $\left\langle\mathcal{G}_{\Phi}, \Omega\right\rangle \models \varphi \mathcal{U} \psi$.

For $\neg(\varphi \mathcal{U} \psi)$ formulas, by the above Proposition 21 and the induction hypothesis there does not exist any finite path $\Omega_{0}, \Omega_{1} \ldots \Omega_{n}$ in $S_{\mathcal{G}_{\Phi}}$ such that $\Omega_{0}=\Omega,\left\langle\mathcal{G}_{\Phi}, \Omega_{n}\right\rangle \models \psi$ and $\left\langle\mathcal{G}_{\Phi}, \Omega_{i}\right\rangle=\varphi$ for every $0 \leq i \leq n-1$. Consequently $\left\langle\mathcal{G}_{\Phi}, \Omega\right\rangle \not \models \varphi \mathcal{U} \psi$ and hence $\left\langle\mathcal{G}_{\Phi}, \Omega\right\rangle \models \neg(\varphi \mathcal{U} \psi)$. 


\subsection{Model Existence and Completeness}

Using the nondeterministic structure $\mathcal{G}_{\Phi}$ (which was defined in the previous subsection), we are now able to build a model of any $\mathcal{F C}$-consistent set.

Lemma 25. (Path Existence) For every $\Omega \in S_{\mathcal{G}_{\Phi}}$ there exists at least one infinite fulfilling path $\pi=\Omega_{0}, \Omega_{1}, \ldots$ where $\Omega_{0}=\Omega$.

Proof. Let us show how to build the path $\pi$ depending on the mscc to which $\Omega$ belongs. If $[\Omega]$ is a self-fulfilling mscc, then choose $\pi^{\prime}$ to be any finite path that covers all the states in $[\Omega]$. Then, the infinite path $\pi=\pi^{\prime}, \pi^{\prime}, \pi^{\prime}, \ldots$ is fulfilling. Otherwise, if $[\Omega]$ is not a self-fulfilling mscc, by Corollary 23, there exists $\Omega^{\prime} \in S_{\mathcal{G}_{\Phi}}$ such that $\Omega^{\prime} \notin[\Omega]$, $\Omega^{\prime} \in R_{\mathcal{G}_{\Phi}}^{+}(\Omega)$ and $\left[\Omega^{\prime}\right]$ is a self-fulfilling mscc. Let $\pi_{1}$ be any finite path from $\Omega$ to $\Omega^{\prime}$ and let $\pi_{2}$ be the infinite path in $\left[\Omega^{\prime}\right]$ constructed as in the previous case. Then, $\pi=\pi_{1}, \pi_{2}$ is an infinite fulfilling path.

Lemma 26. (Model Existence) Let $\pi=\Omega_{0}, \Omega_{1} \ldots$ an infinite fulfilling path in $S_{\mathcal{G}_{\Phi}}$. Then, the PLTL-structure $\mathcal{M}_{\pi}$ defined by

$$
\begin{aligned}
& \quad-S_{\mathcal{M}_{\pi}}=\Omega_{0}, \Omega_{1}, \ldots \\
& \quad-V_{\mathcal{M}_{\pi}}\left(\Omega_{i}\right)=\left\{p \mid p \in \Omega_{i}\right\} \\
& \text { satisfies that }\left\langle\mathcal{M}_{\pi}, i\right\rangle \models \varphi \text { for every } i \in \mathbb{N} \text { and every } \varphi \in \Omega_{i} \text {. }
\end{aligned}
$$

Proof. Immediate consequence of Lemma 24.

Finally, we are able to prove the completeness of $\mathcal{F C}$.

Theorem 27. (Completeness of $\mathcal{F C}$ ) For any set of formulas $\Gamma \cup\{\chi\}$, if $\Gamma \models \chi$ then $\Gamma \vdash_{\mathcal{F C}} \chi$.

Proof. Suppose that $\Gamma \nvdash_{\mathcal{F C}} \chi$. Then, by rule $(C d), \Gamma, \neg \chi \nvdash_{\mathcal{F C}} \mathbf{F}$. Hence, by Corollary 12, Lemma 25 and Lemma 26 there exists a model of $\Gamma \cup\{\neg \chi\}$. Therefore, $\Gamma \not \models \chi$.

\section{Concluding Remarks}

We have introduced a sound and complete (finitary) sequent calculus $\mathcal{F C}$ for the logic PLTL. The calculus $\mathcal{F C}$ is cut-free and invariant-free and it leads to a new deduction style in temporal logic. We are working on the mechanization of the calculus $\mathcal{F C}$ in the generic proof-assistant Isabelle (cf. http://isabelle.in.tum.de) in order to allow the interactive formalization of $\mathcal{F C}$-proofs for temporal properties. Tableaux and resolution methods are better suited for completely automatic theorem proving. In this regard, the rules $(\mathcal{U} L)_{2}$ and $(\diamond L)_{2}$ give rise to new ideas for improving the existing methods of temporal tableaux and temporal resolution. Following these ideas, we are also working on avoiding the construction of the whole states-graph in the tableaux framework and the construction of invariants in the resolution setting. These methods should manage formulas of the form $(\Delta\urcorner \wedge \varphi) \mathcal{U} \psi$ such that $\Delta$ is also part of the set of formulas to be processing. Hence, from the point of view of efficiency, shared formulas would be very useful for practical implementation. Additional future work includes the extension of this ideas to the branching case, the first-order case (in spite of its incompleteness) or its complete fragments. 


\section{References}

1. M. Fisher. A resolution method for temporal logic. In IJCAI, pages 99-104, 1991.

2. D. Gabbay, A. Pnueli, S. Shelah, and J.Stavi. On the temporal analysis of fairness. In POPL '80: Proceedings of the 7th ACM SIGPLAN-SIGACT symposium on Principles of programming languages, pages 163-173, New York, NY, USA, 1980. ACM Press.

3. G. Gentzen. Untersuchungen uber das logische schliessen. In M. E. Szabo, editor, Mathematische Zeitschrift, 39:176-210, 405-431, 1934-35. English translation in "The collected papers of Gerhard Gentzen”, pages 68-131. North-Holland, 1969.

4. J. A. W. Kamp. Tense Logic and the Theory of Linear Order. PhD. Thesis, University of California, Los Angeles, 1968.

5. O. Lichtenstein and A. Pnueli. Propositional temporal logics: Decidability and completeness. Logic Journal of the IGPL, 8(1), 2000.

6. B. Paech. Gentzen-systems for propositional temporal logics. In CSL, pages 240-253, 1988.

7. F. Pfenning. Structural cut elimination: I. intuitionistic and classical logic. Inf. Comput., 157(1-2):84-141, 2000.

8. R. Pliuskevicius. Investigation of finitary calculus for a discrete linear time logic by means of infinitary calculus. In Baltic Computer Science, pages 504-528, 1991.

9. M. Reynolds and C. Dixon. Theorem-proving for discrete temporal logic. In Handbook of Temporal Reasoning in Artificial Intelligence, pages 279-314. Elsevier, 2005.

10. A. P. Sistla and E. M. Clarke. The complexity of propositional linear temporal logics. $J$. ACM, 32(3):733-749, 1985.

11. A. Szalas. Temporal logic of programs: A standard approach. In Time and Logic. A Computational Approach, pages 1-50. UCL Press Ltd., 1995.

12. P. Wolper. Temporal logic can be more expressive. Information and Control, 56(1-2):72-99, 1983. 